$32^{\text {nd }}$ European Modeling \& Simulation Symposium

$17^{\text {th }}$ International Multidisciplinary Modeling \& Simulation Multiconference

\title{
Creating the Future Airport Passenger Experience: IMHOTEP
}

\section{Miguel Mujica Mota ${ }^{1}$, Paolo Scala ${ }^{2,}{ }^{*}$, Ricardo Herranz ${ }^{3}$, Michael Schultz $^{4}$ and Edgar Jimenez 5}

\author{
${ }^{1}$ Amsterdam University of Applied Sciences - Aviation Academy, Weesperzijde 190, Amsterdam, 1097 DZ, The \\ Netherlands \\ ${ }^{2}$ Amsterdam University of Applied Sciences - Amsterdam School of International Business, Fraijlemaborg 133, \\ Amsterdam, 1102 CV, The Netherlands \\ ${ }^{3}$ Nommon Solutions and Technologies SL, Calle C. Coello 124, Madrid, 28006, Spain \\ 4 Technical University of Dresden - Institute of Logistics and Aviation, Helmholtzstrasse 10, Dresden, 01069, \\ Germany \\ ${ }^{5}$ Cranfield University - Centre for Air Transport Management, College road, Cranfield, MK43 oAL, United \\ Kingdom
}

*Corresponding author. Email address: p.m.scala@hva.nl

\begin{abstract}
As the airport of the future is expected to become a multimodal connection platform, one of the main challenges is to create the conditions for travelers to reach their destination by the most efficient and sustainable combination of modes. This will, furthermore, allow the airport and its surrounding region to make a better use of their resources. In this context, the $\mathrm{H} 2020-$ SESAR-2019-2 funded project IMHOTEP, aims at developing a concept of operations and a set of data analysis methods, predictive models and decision support tools that allow information sharing, common situational awareness and real-time collaborative decision-making between airports and ground transport stakeholders. In this paper, the IMHOTEP concepts are presented. The focus is on the project proposed objectives and methodologies applied. Finally, the project expected results and limitations will be discussed.
\end{abstract}

Keywords: Airport, Ground Transport, Multimodal, Passenger Flows, Collaborative Decision Making

\section{Introduction}

The European high-level vision on transport depicts a passenger-centric system that takes travelers from their origin to their destination in a seamless, efficient, predictable, environmentally-friendly and resilient manner (European Commission, 2011). Achieving this vision calls for enhanced modal integration not only in terms of physical infrastructure, but also of business models, operational processes and information systems. In particular, significant potential remains untapped for improving the quality and resilience of the door-todoor passenger journey and the efficiency of airport operations through information sharing and collaborative decision-making across transport modes. Airport Collaborative Decision-Making (A-

(C) 2020 The Authors. This article is an open access article distributed under the terms and conditions of the Creative Commons Attribution (CC BY-NC-ND) license (https://creativecommons.org/licenses/by-nc-nd/4.0/). 
CDM) (EUROCONTROL, 2016) is enhancing the efficiency of airport operations thanks to information sharing and common situational awareness between airports, airspace users (AUs), ground handlers and air navigation service providers (ANSPs), but the concept has so far focused on aircraft turnaround and predeparture sequencing. Total Airport Management (TAM) (SESAR, 2019) is a more holistic concept that foresees closer integration of landside and airside processes, but the passenger access and egress legs are still absent from the picture. The inclusion of ground transport into TAM collaborative decision-making process has been suggested as a future extension that could enable a better integration between the airport and the ground transport system, especially in situations of disruptions. A key enabler for this enhanced integration is the development of information platforms and services that provide airports and ground transport stakeholders with a common and comprehensive picture of the door-todoor passenger flows. In recent years, the pervasive use of personal mobile devices has opened new opportunities for the collection of high-resolution passenger trajectory data with an unprecedented level of accuracy. These data can in turn be blended with the information available from different stakeholders (e.g., flight departure and arrival information, schedules and travel times of ground access modes, terminal processing times, etc.) not only to monitor passenger flows in real-time, but also to build shortterm predictive models and develop look-ahead strategies. This shared, permanently updated picture of the actual and future status of passenger flows would facilitate coordination between transport modes, as well as the development of passenger information services for the planning and reconfiguration of the door-to-door journey, enhancing the passenger experience and rendering the transport system more resilient against disruptive events.

IMHOTEP aims to seize these opportunities by developing a set of enabling technologies able to provide a holistic view of the airport processes, the ground transport system and the passenger flows, with the ultimate purpose of improving the quality, efficiency and resilience of the door-to-door passenger journey (see Fig. 1).

Besides IMHOTEP, there are other similar projects that address multimodality such as: TRANSIT (https://cordis.europa.eu/project/id/893209), MODUS (https://cordis.europa.eu/project/id/891166) and XTEAM (https://cordis.europa.eu/project/id/891061). In table 1 , it can be found a comparison between the project in terms of scope and area of application. The table highlights the difference between IMHOTEP and the mentioned projects. The scope of IMHOTEP is to use airports and passenger information to enhance the passenger travel experience from door-to-airport gate and vice versa, while the scope of the other project is about the improvement of the air transport within an intermodal context. The area of application of IMHOTEP is bounded among the airport terminal and the ground transport connecting to the airport. The area of application of the other projects entails the whole door-to-door intermodal travel at a higher level, as they have a more strategic focus (e.g. assessment of intermodal concepts on transport system as a whole), while IMHOTEP's objective is to come up with decision support tools for real-time decision making.

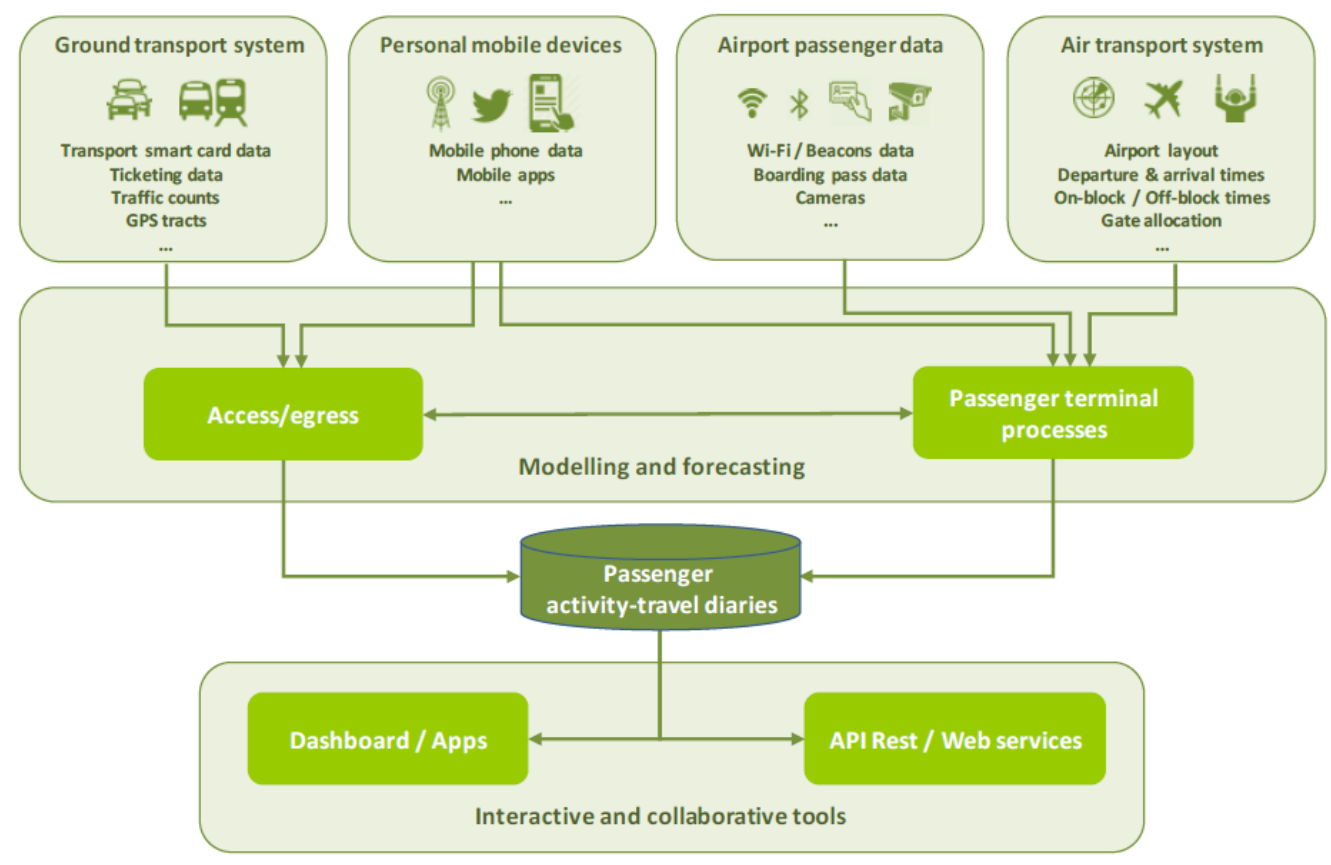

Figure 1. IMHOTEP concept 
In this paper, the IMHOTEP project is discussed with emphasis on the methodology applied, which comprises the use of novel algorithms for passenger flow management along their door-to-door journey and simulation models that will test, calibrate and validate these algorithms. In particular, this paper pays attention to the simulation part, by describing how the simulation models are conceptualized. The simulation part, as it will be described in more details in the next sections, comprises two models, one for the airport access/egress, and another for the airport terminal. The two models will be integrated into one for simulating the entire passenger door-to-door travel experience.

Table 1. Comparison between IMHOTEP and similar projects

\begin{tabular}{|c|c|c|}
\hline Project & Scope & Area of application \\
\hline IMHOTEP & $\begin{array}{l}\text { Integration of } \\
\text { information between } \\
\text { airport and ground } \\
\text { transport stakeholders } \\
\text { for a better coordination } \\
\text { of operations. }\end{array}$ & $\begin{array}{l}\text { Airport terminal operations } \\
\text { and airport external ground } \\
\text { transportation operations. }\end{array}$ \\
\hline TRANSIT & $\begin{array}{l}\text { Design a managing } \\
\text { system for seamlessly } \\
\text { integrated intermodal } \\
\text { transport. }\end{array}$ & $\begin{array}{l}\text { Intermodal transportation } \\
\text { operations. }\end{array}$ \\
\hline MODUS & $\begin{array}{l}\text { Assessing the role of air } \\
\text { transport within an } \\
\text { integrated, intermodal } \\
\text { approach. }\end{array}$ & $\begin{array}{l}\text { Intermodal transportation } \\
\text { operations. }\end{array}$ \\
\hline X-TEAM & $\begin{array}{l}\text { Integrating air transport } \\
\text { with intermodal } \\
\text { network to enable the } \\
\text { door-to-door } \\
\text { connectivity, up to } 4 \\
\text { hours, between any } \\
\text { location in Europe. }\end{array}$ & $\begin{array}{l}\text { Intermodal transportation } \\
\text { operations. }\end{array}$ \\
\hline
\end{tabular}

Below the main contributions of the paper are listed:

- First, the integration of the two models represents a key contribution into the field, as most research has focused only on the passenger flow within terminals, and not the entire passenger's doorto-door journey.

- Second, this paper proposes a framework for developing the two integrated models, pointing out the requirements both from a technical and operational point of view.

- Third, the challenges (technical and operational) brought by the modeling part will be highlighted, as they represent the starting point for future developments.

The remaining of the paper is as follows: in the next section, the project is described with its operations and objectives. Then, the different methodologies applied are presented, with a focus on the development of the operation at terminal level which will be part of the team that works in the respective work package. Finally, a discussion on the expected results and challenges of the project is given.

\section{IMHOTEP: Project Description}

\subsection{Operations Description}

The backbone of the IMHOTEP concept is the common view of the real-time passenger flows and their shortterm evolution. The way the project proposes to materialize this concept is briefly outlined as follows: Passenger flows will be represented in a disaggregated manner, by means of the concept of Passenger Activity-Travel Diary. A Passenger Activity-Travel Diary will be composed by the sequence of activities and trip stages performed by the passenger. Each activity or stage of the journey will have a start time, a start location, an end time, an end location, and a set of additional attributes selected from a list of predefined options for each type of activity or stage (e.g., the activity prior to the trip of a departing passenger or the subsequent activity to the trip of an arriving passenger will have a an attribute called 'activity type' that may take different possible values: 'home', 'work', 'leisure', etc.; the access and egress legs will have an attribute called 'transport mode' that may take the values 'rail', 'metro', 'bus', 'private car', 'taxi', etc.). For a more detailed representation of the Passenger Activity-Travel Diary, please refer to Fig. 2.

The main operations related to the Passenger Activity-Related Diary are Departures, Connections and Arrivals, and they are performed in the following environments:

1. Ground access: where passengers start or end their journey (home, work, another place). Transport mode choice (bus, train, private car, taxi).

2. Airport landside: airport entries/egresses; checkin desks; passengers transit areas.

3. Airport airside: security check area; passport control area; shopping area; gate area; luggage belt area.

4. Air: passengers travelling, inside an aircraft, from origin to destination.

\subsection{Objectives}

The specific objectives of the project are the following:

- Propose a concept of operations for the extension of airport collaborative decision-making to ground transport stakeholders, including local transport authorities, traffic agencies, transport operators and mobility service providers.

- Develop new data collection, analysis and fusion methods able to provide a comprehensive view of the door-to-door passenger trajectory through the coherent integration of different types of high-resolution passenger movement data collected from personal mobile devices and digital 
sensors.

- Develop predictive models and decision support tools able to anticipate the evolution of an airport's passenger flows within the day of operations and assess the operational impact on both airport processes and the ground transport system, with the aim of enabling real-time collaborative decision-making between airports and ground transport stakeholders and enhanced passenger information services.

- Validate the proposed concept and the newly developed methods and tools through a set of case studies conducted in direct collaboration with airports, local transport authorities and transport operators.

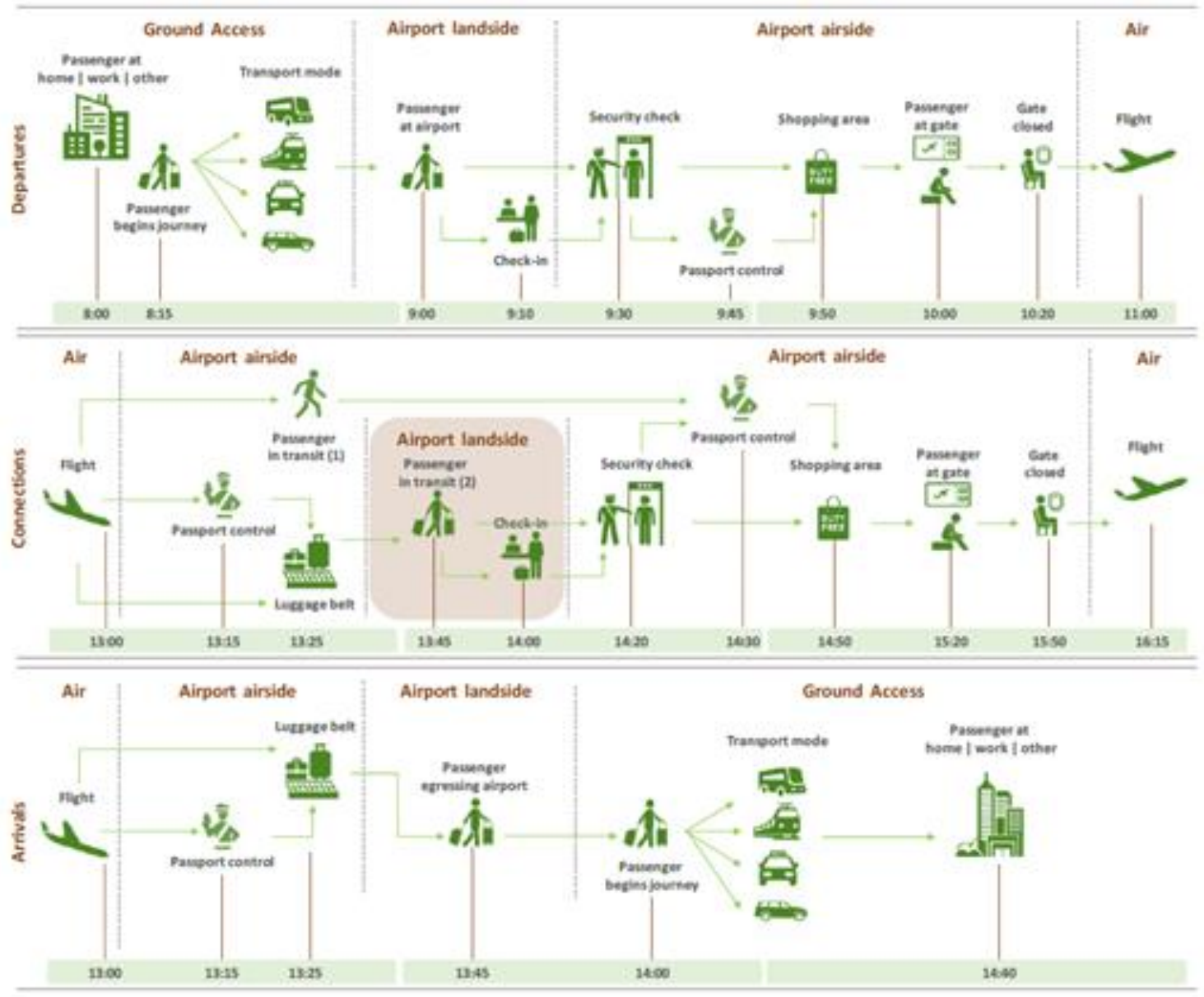

Figure 2. IMHOTEP Operations

\section{Methodology Applied}

The proposed research methodology comprises five main stages. (i) the development of an initial version of the IMHOTEP Concept of Operations (ConOps) and defining the set of case studies that will be used to develop and evaluate the proposed concept; (ii) the collection and preparation of the datasets required for the case studies; (iii) the development of the new data analysis algorithms and the predictive models for the real-time characterization and short-term forecasting of passenger itineraries; (iv) the new data analysis algorithms and predictive models will be then integrated into a prototype decision support tool for collaborative decision-making between airports and ground transport modes; (v) demonstration and evaluation of the proposed concept through a set of case studies. In this paper, we will focus on the third stage, in particular, in the development of the simulation model of the passenger terminal.

\subsection{Development of the IMHOTEP ConOps and Definition of the Case Studies}

The IMHOTEP ConOps aims at extending the A-CDM process to include airport access modes. The ConOps will describe the characteristics of the proposed system from the point of view of airports and ground transport stakeholders, through a set of use cases. The development of the ConOps will encompass the following steps:

- analysis of the current trends in airport multimodal connectivity and identification of new 
intermodal concepts based on information sharing and collaborative decision-making between the airport and ground transport modes.

- identification of user needs and constraints.

- translation of user needs into high-level operational and technical requirements, including the definition of the system architecture, the main interfaces and information flows, the KPIs on which the collaborative decisions will be based, and the required technical capabilities.

- requirement validation. Once the ConOps is defined, the case studies that will be used for the evaluation of the proposed system will take place.

- the input data required for the implementation of the case studies.

\subsection{Data Collection and Preparation}

The data sources used in the project include:

- Anonymized mobile phone records. The position of each user is recorded every 10-20 minutes and is given by the network cell where the user is located. The data also include sociodemographic information of the users, such as age and gender.

- Data on the ground transport system, including the road and rail networks, the available transport services, public transport ticketing and smart card data and road traffic counts, among others.

- Airport data. These data will include data on the airport systems and processes (e.g., airport layout, flight schedules, gate allocation, etc.), aggregated data on the use of airport facilities (e.g., data on the use of the parking facilities, passenger flows at the check-in counters, passport control and security checkpoints, etc.), and disaggregated data on the passenger itineraries in the terminal generated by airport sensors, such as boarding pass scanners and Wi-Fi sensors.

\subsection{Modeling and Short-term Forecasting of Passenger Flows}

The development of new data analysis algorithms and predictive models for the real-time reconstruction and short-term forecasting of passenger flows comprise two activities that will run in parallel: the modelling of the passenger terminal flows and the modelling of the access and egress legs.

\subsubsection{Modeling of Passenger Terminal Process}

The modelling of the passenger terminal processes will encompass two main steps as depicted in Figure 3.

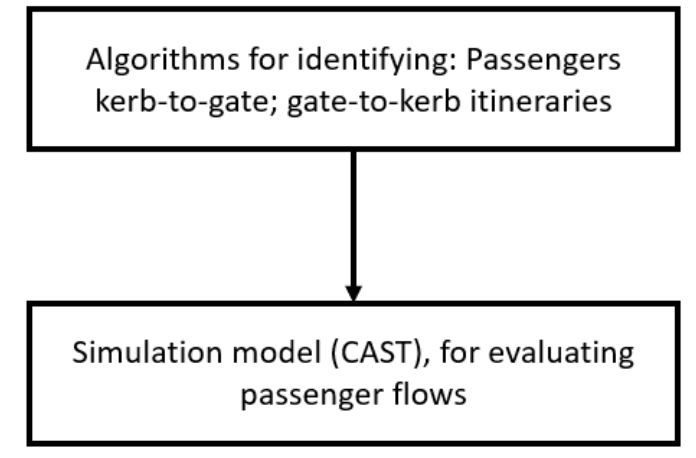

Figure 3. Steps for modeling the passenger terminal process

First, a set of algorithms will be developed for the detailed reconstruction of the passengers' kerb-togate and gate-to-kerb itineraries. Second, a simulation model based on the airport simulation software CAST (ARC, 2020), will be developed. CAST software is a multi-agent simulation framework which allows the modelling of pedestrian, vehicle and aircraft traffic and of landside and airside processes.

The airport agent will be designed to provide, collect, and react on the information included in the Passenger Activity-Travel Diaries. Based on this new situational awareness, the airport system will be dynamically controlled to optimize the utilization of infrastructure and resources in real-time, with the aim of increasing operational efficiency and passenger comfort. A more detailed description of the simulation model of the airport terminal processes will be given in section 4.2.

\subsubsection{Modeling of Passenger Access and Egress}

The modelling of the airport access and egress legs will also comprise two steps (see Fig. 4).

First, we will develop algorithms for the detailed reconstruction of the passengers' door-to-kerb and kerb-to-door itineraries. Particular attention will be paid to the characterization of the different types of passengers (e.g., business travelers vs tourists), as they have very different impacts on the handling process. In a second step, the information about passengers' airport access behavior will be combined with the information on the supply of transport services to/from the airport to build and calibrate a multimodal transport model based on the traffic simulation software Aimsun Live (Aimsun, 2020).

The full door-to-kerb and kerb-to-door trajectories of the passengers will be modelled, in order to generate the access or egress segments of the Passenger Activity-Travel Diaries. The model will be coupled with an emissions model to estimate the environmental impact of the airport landside access. Ground transport modes will be provided with the capability to dynamically re-plan their services based on the information provided by the Passenger Activity-Travel Diaries. 
Algorithms for identifying: Passengers

kerb-to-door; door-to-kerb itineraries

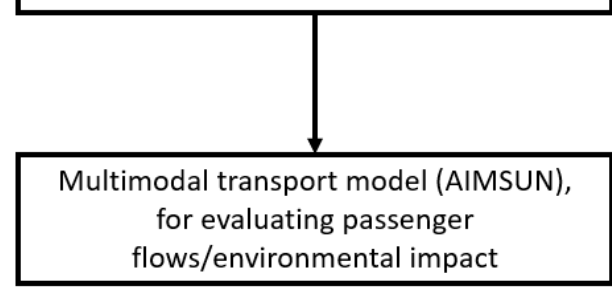

Figure 4. Steps for modeling the access-egress process

\subsection{Development of Decision Support Toolset}

Once the models of the passenger terminal flows and of the access and egress legs are developed, they will be integrated in order to provide a comprehensive view of the full Passenger Activity-Travel Diaries. The integration will involve three main activities:

- the development of the required interfaces so that each model can take as inputs some of the outputs produced by the other models.

- the implementation of the KPIs on which the collaborative decisions will be based. The set of KPIs, specified in the IMHOTEP ConOps, will measure the quality, sustainability, efficiency and resilience of the passenger journey.

- a prototype dashboard will be developed to allow the users to filter, visualize and analyze the KPIs of their interest according to different criteria.

\subsection{Demonstration and Evaluation}

The last stage of the project will be devoted to the demonstration, validation and evaluation of the IMHOTEP concept and the newly developed tools in collaboration with the partner airports and transport operators, as well as with other stakeholders. The evaluation process will include the following steps:

- revise and specify in more detail the case studies defined in the first stage of the project in the light of the results of the data analysis and modelling tasks.

- execute a set of simulation experiments to validate the predictive capabilities of the models.

- emulate the real-time operation of the decision support tools in several working sessions with relevant stakeholders in order to assess the potential of the new tools to support collaborative decision-making.

- assess the performance impact of the proposed concept along different dimensions, with particular focus on the benefits for the passenger, but also looking at the efficiency in the use of airport resources and the sustainability of the airport access modes.
Once the evaluation is completed, the results of the case studies will be complemented with a feasibility analysis of the proposed concept. The knowledge extracted from the case studies and the feasibility study will be synthesized into a set of guidelines and recommendations on the applicability of the IMHOTEP $\mathrm{C}$

\section{Passenger Terminal Process Simulation}

The goal of the passenger terminal process simulation model is to develop and calibrate a modeling tool that reconstructs and simulates the flows of passengers inside the airport terminal. In this section, a brief state of the art regarding the modeling of airport terminal, and a description of the simulation model developed are given.

\subsection{State of the Art regarding the Simulation of Airport Terminals}

The most commonly used paradigms for the simulation of passenger terminal flows are discrete event simulation and agent-based modelling. Discrete event simulation has been used to analyze the times spent at each stage of the compulsory activities at the airport (e.g., security check), identify bottlenecks, and design possible strategies to minimize these times (Alodhaibi et al., 2017; Rausch and Kljajić, 2006). Agent-based modelling provides a more detailed representation of the individual passenger trajectories, allowing the study of how the passengers move inside the airport, including discretionary activities (e.g., shopping), and the analysis of how the visited locations and the time spent at each of them varies with personal preferences, trip purposes, etc. Relevant examples can be found in Schultz et al. (2011), where agent-based modelling is used to study the passenger handling processes and test the impact of adaptive airport signage, and in Ma et al. (2011), where an agent-based model is used to evaluate the level of use of different airport facilities; the probability that a passenger chooses a certain activity is modelled as a function of the passenger characteristics (sociodemographic profile, number of bags, etc.), the available time, and a walking distance threshold. Outside academia, there exists commercial software for the microscopic simulation of the passenger movements, such as CAST.

\subsection{Development of the Passenger Terminal Simulation Model}

The simulation model for the airport passenger terminal, will be developed by following the steps: model specifications; model data analysis; model design and development; model validation. Figure 5 shows how these steps are linked together.

\subsubsection{Model Specifications}

The technical specification of the model of the passenger terminal processes, will be developed 
starting from the high-level system specification provided by the IMHOTEP ConOps.

The passenger terminal model will include the handling processes of passenger arrival:

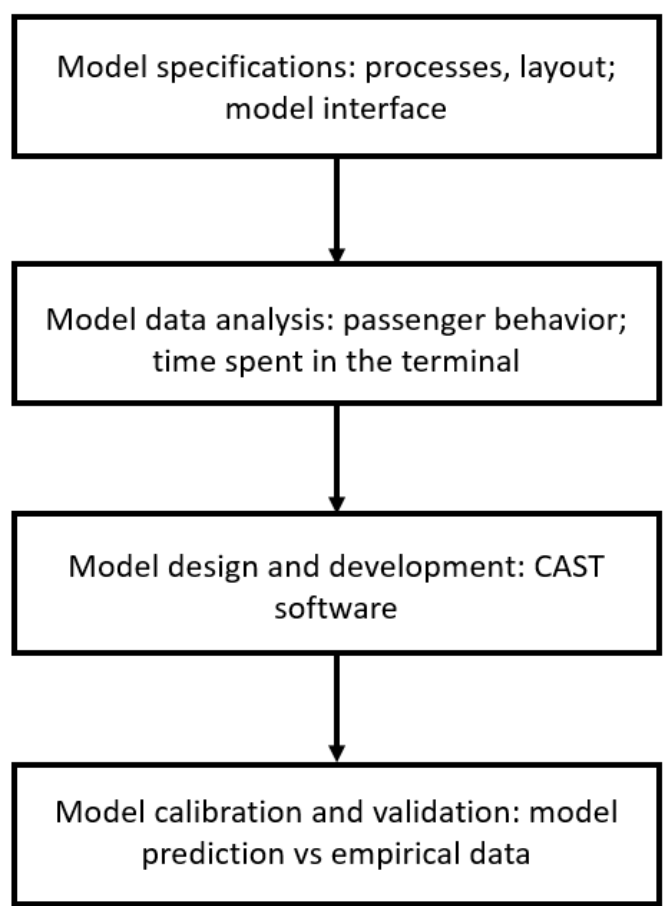

Figure 5. Steps for developing the airport passenger terminal simulation model

In the following subsection, each step is discussed.

- Check-in.

- Border control.

- Security.

- Boarding pass control.

and departure:

- Deboarding.

- Border control.

- Baggage handling.

- Custom.

The layout and amount of facilities of the airport terminal will be provided by the airport operators that will cooperate in the project.

The specification will also include the interface requirements for the integration of the passenger terminal model with the access-egress model (see section 3.3.2).

\subsubsection{Model Data Analysis}

A set of data fusion and analysis methodologies aimed at reconstructing the passenger behavior inside the terminal will be derived from a variety of data generated by personal mobile devices and airport sensors (mobile phone records, Wi-Fi sensors, boarding pass scanners, etc.). With the development of algorithms, the type of passenger (business traveler, tourist, etc.) will be identified and the time spent by different types of passengers at the different terminal processes as well as in other discretionary activities (e.g., shopping) will be measured. The data from personal mobile devices will also be blended with other data (e.g., airport surveys) to enrich the characterization of the passengers' sociodemographic profile and other relevant factors that may explain the passenger behavior inside the terminal.

\subsubsection{Model Design and Development}

To develop an airport terminal simulation model, incorporating the extended $\mathrm{A}-\mathrm{CDM}$ mechanisms defined in the IMHOTEP ConOps, a simulation model of the terminal passenger flows will be developed using CAST multi-agent airport simulation software. The model will include the airport layout, the airport processes, and the passengers' behavior. CAST terminal software allows to model airport terminal processes and to obtain a clear and detailed representation of the them, as they appear in real world airport terminals. A snapshot of the animation of CAST terminal model can be seen in Figure 6. The model will include the necessary mechanisms to simulate the airport decision-making processes considered in the IMHOTEP ConOps (e.g., dynamic assignment of airport resources based on the shortterm forecast of terminal passenger flows).

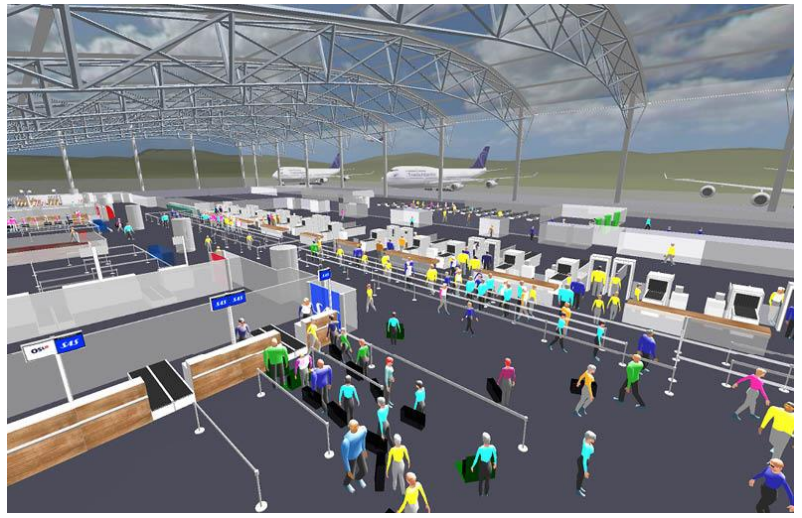

Figure 6. CAST terminal model animation. Source: https://arc.de/cast-terminal-simulation/

\subsubsection{Model Calibration and Validation}

The simulation model will be calibrated and validated by comparing the model results with the information measured by means of the algorithms as mentioned in section 4.2.2. The model will be verified and validated based on diverse statistical techniques making an intensive use of the datasets from the project. Then the results of the statistical analysis will be translated into empirical probability distributions of the passenger processes. Diverse techniques from Machine learning or data mining will be used for 
predicting the behavior of the passengers within the terminal.

\section{Contribution and Challenges of the IMHOTEP Project}

The results of IMHOTEP are expected to set the path for the development of a multimodal airport collaborative decision-making environment. Beyond the A-CDM context, the new data analysis algorithms and the simulation solutions developed by IMHOTEP could be the basis for new solutions for the planning and management of transport systems. Airport managers are one of the stakeholders that will benefit from the outcome of the IMHOTEP project, as they will be able to make more accurate and reliable decisions. The integration of the data between ground transport and airports will enhance the accuracy in the flow of passengers in and out of the airport, and consequently both airports and ground transport can make a better planning of their resources. IMHOTEP itself will reveal the potential improvement of performance once information is shared not only with the stakeholders of the system itself but with the users of the systems. Furthermore, IMHOTEP will enable the passengers to perform a self-regulated operation as they will be able to perceive, assess and make informed decisions based on the information sources of the system. This approach has the potential to give way to new types of systems where transparency and decentralized decision making are the key characteristics; in other words, IMHOTEP will reveal the potential of new paradigms of systems management.

The use of simulation allows to evaluate the potential of information sharing and also different policies and scenarios to validate the overall IMHOTEP ConOps, however, it comes with some challenges:

1. Validation. The validity of the simulation model outcome relies on the data collected about airport process specifications, provided by the airport operator, and the data on passenger behavior, collected by using passengers' personal mobiles and airport sensors. Therefore, the data should be reliable and available and in a form that is useful for the validation purposes.

2. Integration of multi-model approach. The different models of IMHOTEP need to be integrated to interchange information amongst them. This will be a challenge as it is foreseen a large-scale model.

3. Personal data protection, as the proposed concept relies on the anonymization of different personal data, adequate data security policies and measures ensuring compliance with GDPR are required.

All these challenges require a particular effort from a modeling point of view, especially regarding the integration of the different models. Running the models on-line or off-line is a critical decision that the modelers need to make, which impacts the way how the two models will communicate (via a shared database or a synchronous exchange of data).

\section{Funding}

This project has received funding from the SESAR/European Union's Horizon 2020 research and innovation program under grant agreement No 891287. The opinions expressed herein reflect the authors' view only. Under no circumstances shall the SESAR Joint Undertaking is responsible for any use that may be made of the information contained herein.

\section{Acknowledgements}

The authors would like to thank the Amsterdam University of Applied Sciences for the support in this research, as well as the Dutch Benelux Simulation Society (www.dutchbss.org) and EUROSIM (www.eurosim.info, www.eurosim2022.eu) for disseminating the results of this work.

\section{References}

Aimsun, 2020. Aimsun Live. https://www.aimsun.com/aimsun-live/

Alodhaibi, S., Burdett, R. L., \& Yarlagadda, P. K. (2017). Framework for airport outbound passenger flow modelling. Procedia Engineering, 174, 1100-1109.

ARC, 2020. CAST. https://arc.de/cast-simulationsoftware/

EUROCONTROL, 2016. A-CDM Impact Assessment Final Report.

European Commission, 2011. White Paper: Roadmap to a Single European Transport Area - Towards a competitive and resource efficient transport system. $\operatorname{COM}(2011) 144$ final.

Ma, W., Kleinschmidt, T., Fookes, C., \& Yarlagadda, P. K. (2011). Check-in processing: simulation of passengers with advanced traits. In Proceedings of the Winter Simulation Conference (pp. 1783-1794): Winter Simulation Conference..

Rauch, R., \& Kljajić, M. (2006). Discrete event passenger flow simulation model for an airport terminal capacity analysis. Organizacija, 39(10).

Schultz, M., \& Fricke, H. (2011). Managing Passenger Handling at Airport Terminal Individual-based Model for Stochastic Passenger Behavior. 9th USA/Europe Air Traffic Management Research and Development Seminar, Berlin, Germany.

SESAR, 2019. Total Airport Management. https://www.sesarju.eu/projects/tam 


\section{Cranfield University}

2020-09-18

\section{Creating the future airport passenger experience: IMHOTEP}

Mota, Miguel Mujica

Caltek

Mujica Mota M, Scala P, Herranz R, et al., (2020) Creating the future airport passenger experience: IMHOTEP. In: European Modeling \& Simulation Symposium, 16-18 September 2020,Online https://doi.org/10.46354/i3m.2020.emss.024

Downloaded from Cranfield Library Services E-Repository 